\title{
Local Control Theory using Trajectory Surface Hopping and Linear-Response Time-Dependent Density Functional Theory
}

\author{
Basile F. E. Curchod ${ }^{\S a}$, Thomas J. Penfoldabc, Ursula Rothlisberger ${ }^{a}$, and Ivano Tavernelli ${ }^{\star a}$ \\ §SCS-Metrohm Foundation Award for best oral presentation
}

\begin{abstract}
The implementation of local control theory using nonadiabatic molecular dynamics within the framework of linear-response time-dependent density functional theory is discussed. The method is applied to study the photoexcitation of lithium fluoride, for which we demonstrate that this approach can efficiently generate a pulse, on-the-fly, able to control the population transfer between two selected electronic states. Analysis of the computed control pulse yields insights into the photophysics of the process identifying the relevant frequencies associated to the curvature of the initial and final state potential energy curves and their energy differences. The limitations inherent to the use of the trajectory surface hopping approach are also discussed.
\end{abstract}

Keywords: Born-Oppenheimer approximation - Linear-response time-dependent density functional theory . Local control theory $\cdot$ Nonadiabatic dynamics

\section{Introduction}

Through the interaction with an external perturbing field, molecular systems can be electronically promoted into an excited state. The ensuing dynamics, which is often characterized by the breakdown of the Born-Oppenheimer approximation through the action of sizeable nonadiabatic couplings between electronic states, play an important role for a wide range of applications. To understand the dynamics of these ultrafast relaxation processes in molecules, nonadiabatic molecular dynamics (MD) schemes, such as the widely used Tully's Fewest Switches Trajectory Surface Hopping (TSH), ${ }^{[1]}$ constitute a valid approach because it allows for an unbiased investigation of systems with a

\footnotetext{
${ }^{*}$ Correspondence: Dr. MER I. Tavernellia Tel.: +41216930328

E-mail: ivano.tavernelli@epfl.ch

aLaboratory of Computational Chemistry and Biochemistry

Ecole Polytechnique Fédérale de Lausanne, $\mathrm{CH}-1015$ Lausanne

baboratory of Ultrafast Spectroscopy

Ecole Polytechnique Fédérale de Lausanne,

$\mathrm{CH}-1015$ Lausanne,

cSwissFEL

Paul Scherrer Institute

$\mathrm{CH}-5232$ Villigen
}

large number of degrees of freedom. In this approach, the nuclear wavepacket is discretized into a swarm of independent classical trajectories and propagated independently according to the classical Newton equation. Although this neglects all nuclear quantum correlation effects, the ability of this approach to accurately describe nonadiabatic dynamics has led to it becoming a widely used approach with successful applications to a range of problems. ${ }^{[2]}$

In contrast to nuclear wavepacket propagation schemes, ${ }^{[3]}$ the spatial locality of the potential governing the time-evolution of the trajectories makes these approaches ideal for an on-the-fly implementation in an $a b$ initio molecular dynamics scheme since, at any instant of time, energies and nuclear forces are only required at a single position in configuration space. As a consequence, TSH has been coupled with a variety of electronic structure methods. ${ }^{4]}$ Important in the context of this work is its implementation within the LinearResponse Time-Dependent Density Functional Theory[5] (LR-TDDFT) framework, which makes the simulation of medium to large systems possible.[6]

For systems exposed to external perturbations, simulations are usually performed without the explicit inclusion of the (timedependent) interaction between the perturbing field and the system of interest. In most cases, the simulations are started in the Franck-Condon region after an ad hoc excitation to a resonant state. However for the retrieval of fine spectroscopic details it can be important to explicitly study the interaction between the perturbing field and the dynamics of the system in question. Beyond this, by modulating the properties of the applied field it is possible to selectively drive the system into a region of interest in configuration space, yielding a control mechanism that may be used for a wide variety of applications, including the selection of specific photochemical products and the generation of molecules in predefined excited states for subsequent use in experiments (see for example ref. [7]).

Coherent control of molecular systems, pioneered in the mid-1980s by the Tannor-Rice pump-dump ${ }^{[8]}$ and the Brumer-Shapiro ${ }^{[9]}$ schemes, is most commonly simulated using optimal control theory (OCT). ${ }^{[10]}$ Here, the external field is optimized using a variational principle combined to an iterative process, which requires forward and backward propagations in time. Despite its success and its close analogy to the learning algorithm used experimentally, the repeated propagation of the system back and forth in time makes it computationally extremely expensive. Alternatively, we have recently implemented an approach ${ }^{[11]}$ based upon Local Control Theory (LCT). ${ }^{[12]}$ In contrast to OCT, LCT is based on the dynamics of a defined target (a quantum mechanical expectation value) and a control pulse is 
calculated on-the-fly (using only instantaneous or local properties of the dynamics), so that to ensure the desired increase (or target quantity at each instant of time. As the shaped electric field is calculated and applied on-the-fly, this approach is wellsuited for $a b$ initio molecular dynamics because the electronic energies, nuclear dynamics and effect of the external perturbation can all be obtained from a single nuclear propagation.

In this article, we briefly review the basic ideas of LCT within the framework of trajectory surface hopping ab initio molecular dynamics, which is then applied to study the controlled photoexcitaiton of LiF. We show that LCT is able to efficiently control this photoexcitation process, and that the calculated control pulse contains interesting information about the shape of the potential energy curves and about the nuclear motion contained within the dynamics. Finally, we highlight characteristics of the pulse that arise from the approximations used within surface hopping molecular dynamics, in particular the neglect of correlation between the trajectories and the lack of decoherence of the complex amplitudes along a single trajectory.

\section{Theory}

TSH portrays the nonadiabatic dynamics of a nuclear wavepacket as a swarm of independent classical trajectories, each having the possibility to hop between electronic states according to Tully's surface hopping switching probability. ${ }^{[1,4]}$ For any one trajectory within the swarm (labeled with $\alpha$ ) the dynamics is initiated in a given electronic state $(j)$, and the nuclei are evolved in time based on classical forces obtained from the Newton equation of motion (Eqn. (1)) where $E_{j}^{e l, \alpha}$ represents the electronic energy of the molecular system at the position of the classical trajectory $\alpha$, in electronic state $j$ ( $\boldsymbol{F}$ and $\boldsymbol{R}$ are symbols

$$
\boldsymbol{F}_{j}^{\alpha}=-\nabla_{\boldsymbol{R}} E_{j}^{e l, \alpha}
$$
decrease) of the expectation value of the

for collective nuclear forces and coordinates, respectively). The trajectory evolves purely adiabatically with forces evaluated from state $j$. The amount of nonadiabaticity along the nuclear dynamics is monitored by a set of time-dependent complex amplitudes $\left\{C_{j}\right\}$, which evolve according to Eqn. (2). Eqn. (2) is obtained from the time-dependent Schrödinger equation following several approximations (see refs. $[4,13]$ for additional information). At the beginning of the dynamics only the amplitude corresponding to the initial electronic state $\left(C_{j}^{\alpha}\right)$ is non-zero. The complex amplitudes evolve coherently on the support of the classical trajectory, explicitly taking into account the nonadiabatic effects between electronic states $j$ and $i$ through the nonadiabatic coupling vectors $\boldsymbol{d}_{j i}(\dot{\boldsymbol{R}}$ represent nuclear velocities).

After each nuclear time step, once the set of complex amplitudes have been propagated, a probability for the trajectory to jump from its driving state $j$ to another electronic state $i$ is computed according to the Tully's Fewest Switches probability. ${ }^{[1,4]}$ Based on a Metropolis criterion, the hop is then either accepted or rejected. If a hop occurs, the classical trajectory will be transferred to the new state and propagated using the forces from this new state. The different photoproducts associated to the nonradiative deexcitation of the molecular system are obtained by collecting the timeevolution of a large number of trajectories.

Recently, we have extended the TSH algorithm to explicitly incorporate the effect of an external electric field within LRTDDFT. ${ }^{[14]}$ An electric field $\underline{\boldsymbol{E}}(t)$ is coupled to the molecular transition dipole moments $\mu_{i j}$ in the equations of motion for the complex amplitudes which allows amplitude to be transferred between electronic states due the effect of a laser pulse for example (Eqn. (3)).

In this way, the dynamics can therefore be initialized in the ground state of the molecular system and the coupling between the molecular system and the applied electric field can directly lead to the population of different electronic states (transfer of amplitude) eventually resulting in a trajectory hop (transfer of trajectory). The intent of this work is to shape an electric field such that it can promote selectively

$$
\begin{aligned}
i \hbar \dot{C}_{j}^{\alpha}(t) & =\sum_{i} C_{i}^{\alpha}(t)\left[E_{i}^{e l, \alpha} \delta_{j i}-i \hbar \dot{\boldsymbol{R}}^{\alpha} \cdot \boldsymbol{d}_{j i}^{\alpha}\right] \cdot \\
i \hbar \dot{C}_{j}^{\alpha}(t) & =\sum_{i} C_{i}^{\alpha}(t)\left[E_{i}^{e l, \alpha} \delta_{j i}-i \hbar \dot{\boldsymbol{R}}^{\alpha} \cdot \boldsymbol{d}_{j i}^{\alpha}-\underline{\boldsymbol{\mu}}_{j i}^{\alpha} \cdot \underline{\boldsymbol{E}}(t)\right],
\end{aligned}
$$

$$
\underline{\boldsymbol{E}}_{[i]}^{\alpha}(t)= \pm \lambda \sum_{j} \Im\left[C_{i}^{\alpha *}(t) C_{j}^{\alpha}(t) \underline{\boldsymbol{\mu}}_{i j}^{\alpha}\right]
$$

the dynamics into a chosen electronic state, which can induce a desired photochemical reaction. Based on LCT, an electric field can be generated and applied on-the-fly along the dynamics, in a way that the population $\left|C_{i}(t)\right|^{2}$ of a given electronic state $i$ is maximized. Such electric field obeys Eqn. (4) at each nuclear time step ${ }^{[11]}$ where only information about the instantaneous state of the molecular system at time $t$ is needed. The additional parameter, $\lambda \in \mathbb{R}$ which is system-dependent, is used to modulate the strength of the control pulse.

\section{Application to Lithium Fluoride}

In a recent work, ${ }^{[11]}$ we have discussed the local control theory applied to lithium fluoride $(\mathrm{LiF})$, where the electric field was shaped to specifically populate the $S_{2}$ state. The electronic energies, nonadiabatic couplings, transition dipole moments, and forces on the nuclei were computed within DFT and LR-TDDFT (within the TammDancoff Approximation, TDA) with the PBE functional. ${ }^{[11]}$ Here, we use the same system but a weaker pulse such that the dynamics of the molecule can be 'recorded' over a larger time period. Obtaining a weaker pulse within LCT is a simple task, which is achieved by decreasing the value of the parameter $\lambda$, here set to 0.05 (it has been shown that the $\lambda$ parameter can strongly affect the underlying control process and the resulting shaped pulse[12]). Two different LCT/TSH trajectories have been calculated, whose initial conditions have been obtained from a Born-Oppenheimer molecular dynamics of $\mathrm{LiF}$ at $150 \mathrm{~K}$ in its electronic ground state.

The first trajectory (Fig. 1) shows a gradual increase of the $S_{2}$ population $\left(\left|\mathrm{C}_{2}(\mathrm{t})\right|^{2}\right)$ due to the applied shaped electric field, and once the population reaches approximately $50 \%$, the trajectory hops from the GS to $\mathrm{S}_{2}$ (after 366fs of dynamics). Following this, the trajectory relaxes in $\mathrm{S}_{2}$ and the shaped pulse exhibits a down-chirping, which corresponds to the decreasing excitation frequency between the GS and $S_{2}$ (Fig. 1, upper panel). This change in the field frequency is due to the underlying classical dynamics of the TSH trajectories and their sudden hops. Hence, taking the Fourier transform of the first part of the LCT pulse until the trajectory hops produces a spectrum dominated by frequencies representative of the dynamics in the Franck-Condon region of the GS (light grey spectra in the inset of Fig. 1). These frequencies correspond to the gap between the GS and $\mathrm{S}_{2}$ along the dynamics. On the other hand, when the overall pulse is Fourier transformed, lower frequencies are observed, which provide information on the relaxation into the $S_{2}$ state. This effect 


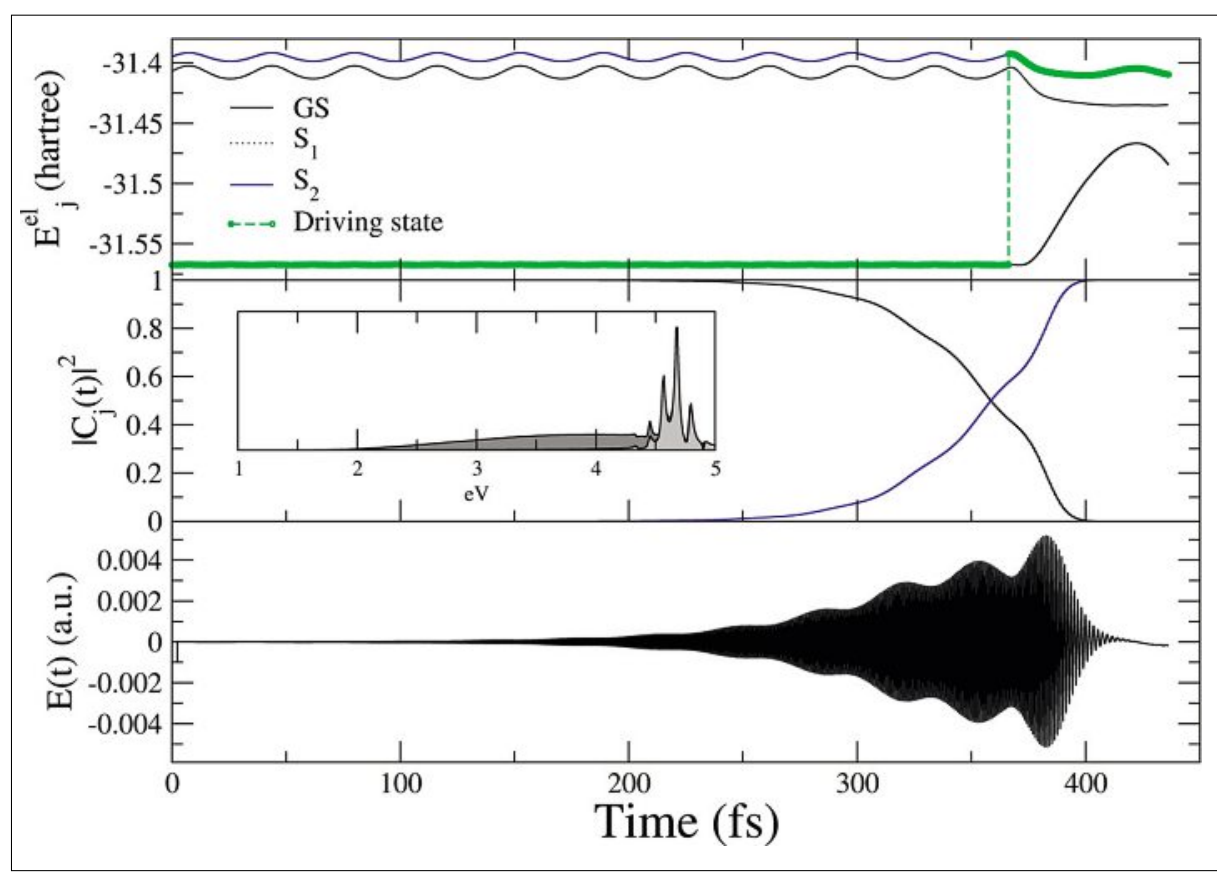

Fig. 1. LCT/TSH of LiF, trajectory 1. Upper panel: Potential energy curves along the dynamics obtained from DFT/PBE and LR-TDDFT/PBE/TDA calculations. Color code: black, GS; dotted black, $\mathrm{S}_{1}$; blue, $\mathrm{S}_{2}$. Green dotted line highlights the running state. Middle panel: Population $\left(|C .(t)|^{2}\right)$ of each electronic state. Inset presents Fourier transforms of the full LCT pulse (dark gray) and of the first part of the pulse, until the trajectory hops (light gray). Lower panel: Calculated LCT pulse in the time domain.

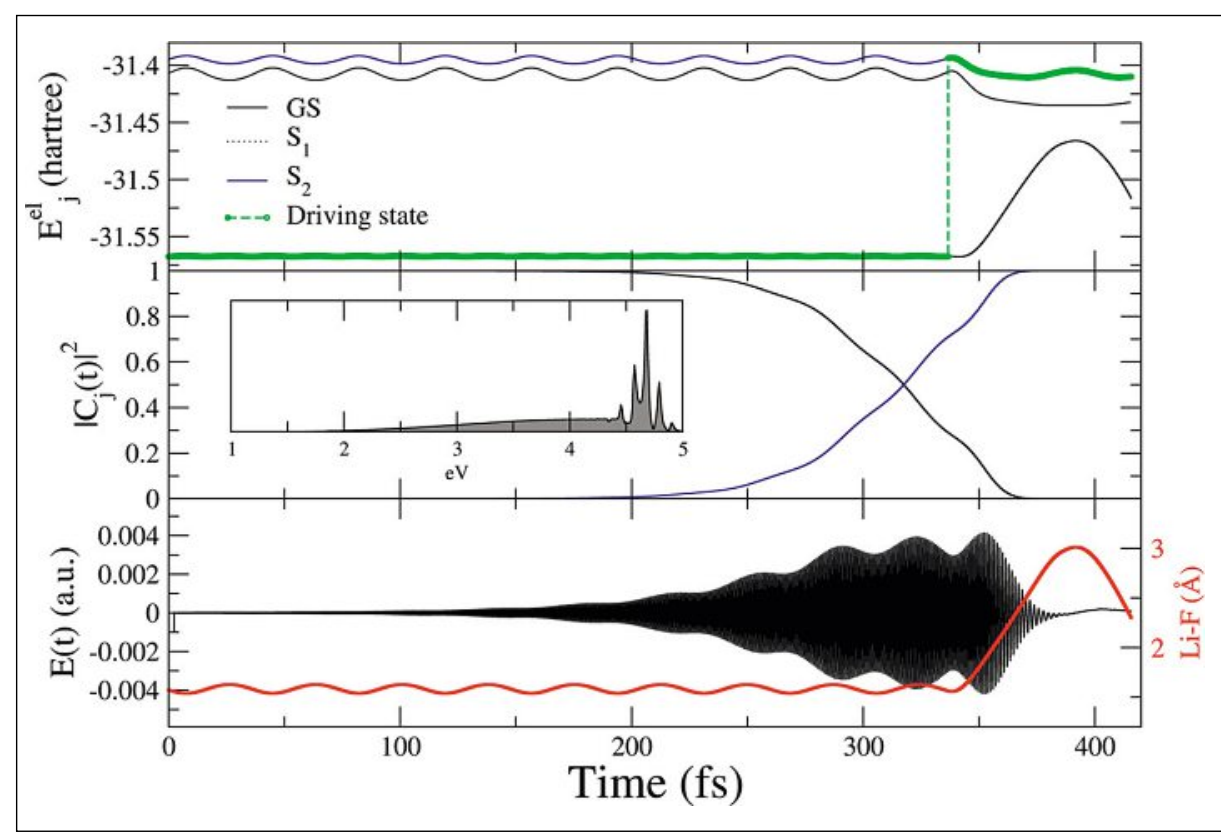

Fig. 2. Same as Fig. 1, but for a second trajectory. Lower panel: the red line corresponds to the LiF bond length along the dynamics.

reflects the fully coherent propagation of the TSH complex amplitudes and the classical dynamics of the TSH trajectories but, importantly, the main physical message is equivalent to that obtained from quantum dynamics simulations (see ref. [11]). The only exception is that in this case the correlation of the nuclear wavepacket implies that the influence of different states dynamics on the spectra of the pulse is spread throughout the entire control pulse. It is also important to point out that when the trajectory jumps to the excited state when only a small amount of amplitude is transferred to the desired electronic state, the shaped pulse will record more information about the driving excited states. On the other hand, when the trajectory jumps at later times (when a large amount of amplitude is already transferred), the field will already be tending to zero and almost no information about the excited state dynamics will be present in the pulse. Therefore, systems with a larger number of degrees of freedom will need more trajectories to characterize all the important frequencies required to successfully design the pulse. In addition, several trajectories are also needed to study the influence of the initial conditions.

Fig. 2 shows the dynamics obtained for a second set of initial conditions. The dynamics is similar to the previous one with the exception that the trajectory jumps at a slightly earlier time. In agreement with the results reported in Fig. 1, the pulse contains a low-frequency oscillation associated to the evolution of the trajectory in the GS, which corresponds to the Li-F bond oscillation (red line in Fig. 2, lower panel). This is in-phase with the envelop oscillation observed in the control pulse that arises because the dipole moment between the GS and $S_{2}$ is largest at the outer turning point of the potential in the ground state (see red line in Fig. 2, lower panel).

\section{Conclusion}

In this article, we have provided an overview of the recently implemented LCT within on-the-fly LR-TDDFT-based nonadiabatic TSH dynamics. LCT uses the instantaneous dynamics of the system to calculate an electric pulse on-the-fly which ensures the desired change of the expectation value of a given operator. This scheme was applied to study the photoexcitation of $\mathrm{LiF}$, for which we target the population of the bound excited state $S_{2}$. The results demonstrate that LCT/TSH can effectively populate the target state and that the resulting control pulse bears information about the overall nuclear dynamics on the initial and final electronic state potentials. Importantly, the profile of the control pulse, and in particular the distinct chirping effect following the hop of the trajectory arises from one of the approximations used within the mixed quantum/ classical scheme surface hopping, i.e. the propagation of classical nuclei with sudden hops between electronic states.

\section{Acknowledgements}

COST action CM0702, Swiss NSF grant 200020-130082, and the NCCR-MUST interdisciplinary research program are acknowledged for funding. BFEC would like to thank Philipp Marquetand for stimulating discussions.

Received: March 22, 2013

[1] J. C. Tully, J. Chem. Phys. 1990, 93, 1061.

[2] E. Tapavicza, I. Tavernelli, U. Rothlisberger, C. Filippi, M. E. Casida, J. Chem. Phys. 2008, 129, 124108; R. Mitric, V. Bonacic-Koutecky, J. Pittner, H. Lischka, J. Chem. Phys. 2006, 125, 024303; M. Barbatti H. Lischka, J. Phys. Chem. A 2007, 111, 2852; M. Vazdar, M. Eckert- 
Maksic, M. Barbatti, H. Lischka, Mol. Phys. 2009, 107, 845; U. Werner, R. Mitric, T. Suzuki, V. Bonacic-Koutecky, Chem. Phys. 2008, 349, 319.

[3] M. H. Beck, A. Jäckle, G. A. Worth, H. D. Meyer, Phys. Rep. 2000, 324, 1.

[4] M. Barbatti, WIREs Comput. Mol. Sci. 2011, 1, 620.

[5] E. Runge, E. K. U. Gross, Phys. Rev. Lett. 1984, 52, 997; M. E. Casida, in 'Recent Advances in Density Functional Methods', Ed. D. P. Chong, World Scientific, Singapore, 1995, p. 155; M. Petersilka, U. J. Gossmann, E. K. U. Gross, Phys. Rev. Lett. 1996, 76, 1212; H. Appel, E. K. U. Gross, K. Burke, Phys. Rev. Lett. 2003, 90, 043005

[6] E. Tapavicza, I. Tavernelli, U. Rothlisberger, Phys. Rev. Lett. 2007, 98, 023001; I. Tavernelli,
E. Tapavicza, U. Rothlisberger, J. Chem. Phys. 2009, 130, 124107; I. Tavernelli, E. Tapavicza, U. Rothlisberger, J. Mol. Struc. (Theochem) 2009, 914, 22; I. Tavernelli, B. F. E. Curchod, U. Rothlisberger, J. Chem. Phys. 2009, 131 196101; I. Tavernelli, B. F. E. Curchod, A. Laktionov, U. Rothlisberger, J. Chem. Phys. 2010, 133, 194104; I. Tavernelli, B. F. E Curchod, U. Rothlisberger, Chem. Phys. 2011, 391, 101.

[7] P. Miéville, S. Jannin, L. Helm, G. Bodenhausen, Chimia 2011, 65, 260.

[8] D. J. Tannor, S. A. Rice, J. Chem. Phys. 1985 83, 5013.

[9] P. Brumer, M. Shapiro, Chem. Phys. Lett. 1986 126, 541.

[10] A. P. Peirce, M. A. Dahleh, H. Rabitz, Phys. Rev. A 1988, 37, 4950.
[11] B. F. E. Curchod, T. J. Penfold, U. Rothlisberger, I. Tavernelli, Phys. Rev. A 2011, 84, 042507.

[12] T. J. Penfold, G. A. Worth, C. Meier, Phys. Chem. Chem. Phys. 2010, 12, 15616; G. Worth, C. Sanz, Phys. Chem. Chem. Phys. 2010, 12, 15570; P. Marquetand, C. Meier, V. Engel, $J$. Chem. Phys. 2005, 123, 204320; S. Graefe, V. Engel, Chem. Phys. 2006, 329, 118 ; P. Marquetand, V. Engel, J. Chem. Phys. 2007, 127, 084115; V. Engel, C. Meier, D. J. Tannor, Adv. Chem. Phys. 2009, 141, 29.

[13] B. F. E. Curchod, U. Rothlisberger, I. Tavernelli, Chem. Phys. Chem. 2013, in press.

[14] I. Tavernelli, B. F. E. Curchod, U. Rothlisberger, Phys. Rev. A 2010, 81, 052508. 